\title{
Une autre année endiablée
}

\author{
par Myrella Roy
}

E

ncore une fois en 2009, la Société canadienne des pharmaciens d'hôpitaux $(\mathrm{SCPH})$ a travaillé à un train d'enfer. Notre quête effrénée et néanmoins allègre pour servir nos membres a laissé sur son passage nombre de réalisations aux escales de la valorisation, de la formation, du partage de l'information et de l'élaboration de normes.

L'embauche de Barbara Wells comme coordonnatrice du projet SCPH 2015 a mis le feu aux activités de l'année. Le projet SCPH 2015 est un programme axé sur la qualité qui propose une vision de l'excellence en pratique pharmaceutique en l'an 2015 et qui vise à améliorer les résultats de la pharmacothérapie et la sécurité des soins aux patients (http://www.cshp.ca/ programs/cshp2015/index_e.asp). En collaboration avec le docteur Neil MacKinnon, délégué présidentiel et agent de liaison pour la vision de la SCPH, madame Wells a déjà fait remarquablement avancer le projet en formant un comité directeur, en recrutant des chargés de projet pour chacune des sections de la SCPH, en sondant les gestionnaires de pharmacie hospitalière du Canada à propos de l'atteinte des buts et objectifs du projet SCPH 2015 dans leur établissement, en distribuant les données initiales du projet SCPH 2015 aux lecteurs du Journal canadien de la pharmacie hospitalière (sous forme d'une réimpression du chapitre afférent d'abord paru dans le rapport 2007-2008 sur les pharmacies hospitalières canadiennes que vous pouvez consulter à http://www.lillyhospitalsurvey. ca/hpc2/content/2008_report/scphF.pdf) et en créant un " tableau de concordance " pour lier le projet aux données probantes et à d'autres normes et meilleures pratiques canadiennes.

Au mois d'avril 2009, la nouvelle et brillante plateforme en ligne du Journal canadien de la pharmacie hospitalière (http://www.cjhp-online.ca) s'est allumée en grande première. Grâce à cette plateforme intuitive, les membres de la SCPH peuvent maintenant plus aisément consulter et fouiller les numéros du Journal publiés depuis l'an 2000. Comme offre de lancement, la SCPH accorde le libre accès à tous les numéros actuels et archivés aux non-membres jusqu'en décembre 2010.

Les Publications officielles de la SCPH se sont parées de tous leurs feux en garnissant ses pages de plusieurs documents nouveaux ou révisés (http://www.cshp.ca/productsServices/ officialPublications/type_f.asp). Le Comité directeur des normes de pratique a fait paraître Lignes directrices sur les services d'information pharmaceutique (en collaboration avec le Réseau de spécialistes en pharmacie [RSP] du domaine de l'information pharmacothérapeutique; en cours de traduction), Programme de doctorat professionnel en pharmacie comme niveau d'entrée à la profession au Canada : document d'information sur ses répercussions potentielles sur les services de pharmacie, Énoncé sur le bilan comparatif des médicaments (en collaboration avec le Groupe de travail sur le bilan comparatif des médicaments), Le pharmacien prescripteur : document d'information et Le pharmaciens prescripteur : énoncé (les deux derniers documents rédigés en collaboration avec le Groupe de travail sur le pharmacien prescripteur). Une année des plus prolifiques!

Au terme de plusieurs années de labeur, l'Association des pharmaciens des établissements de santé du Québec et la SCPH étaient rayonnantes d'enthousiasme lorsqu'elles ont lancé la quatrième édition du Guide pratique des soins palliatifs, en versions française et anglaise (http://www.cshp.ca/productsServices/ otherPublications/careBeyondCure_e.asp). Ce guide fort prisé et son récapitulatif des tableaux de référence en format de poche font le point sur les connaissances en gestion de la douleur et des autres symptômes pour le bien des patients qui sont en fin de vie.

En 2008, le Conseil des associés de la SCPH a allumé les chandelles pour célébrer son $40^{\mathrm{e}}$ anniversaire et a rédigé un mémento historique pour souligner ce jalon majeur. La SCPH a instauré le programme des Associés en 1968 afin de saluer les membres de la SCPH qui se sont distingués par leur leadership, leur dévouement et leur engagement exceptionnels envers l'excellence et le progrès de la pratique. Ce titre souligne les efforts assidus et prolongés des membres dans les sphères du service à la Société, des soins aux patients, de l'enseignement ou de la recherche, et de la pratique en pharmacie hospitalière. Le mémento a d'abord été remis aux associés présents à la Conférence sur la pratique professionnelle en 2008 et a ensuite été transmis par courriel à tous les associés. La SCPH l'a subséquemment traduit en français et, en 2009, elle a procuré les versions finales électroniques de facture professionnelle à tous ses membres (http://www.cshp.ca/programs/fellows/anniversary_e.asp).

La campagne médiatique nationale contre l'élargissement du champ d'exercice des pharmaciens, que l'Ontario Medical 
Association (OMA) a menée l'automne dernier, a provoqué des étincelles au sein de la communauté de la santé. La SCPH et sa section de l'Ontario ont répondu aux messages incendiaires dans une lettre adressée à l'OMA, dont copie a été envoyée à l'Ontario Pharmacists' Association et à l'Ontario Hospital Association, et ont vivement conseillé à l'OMA de respecter sa déclaration au Comité permanent de la politique sociale de l'Assemblée législative de l'Ontario, le 20 septembre 2009, selon laquelle « la collaboration entre les professionnels de la santé est le meilleur moyen de consolider et d'améliorer la prestation de soins sécuritaires et de première qualité. "Une autre lettre a communiqué des sentiments semblables au premier ministre et, par copie conforme, à la ministre de la santé et des soins de longue durée de l'Ontario et elle a aussi complimenté le gouvernement de l'Ontario pour avoir voté la Loi de 2009 modifiant la Loi sur les professions de la santé réglementées. Cette nouvelle loi élargit la gamme de services que peuvent offrir les pharmaciens aux Ontariens (http://www.health.gov.on.ca/french/ publicf/legislationf/regulatedf/regulated_health_professionsf.htm 1). Les documents afférents à la réplique de la Société sont affichés sur le site Web de la SCPH à http://www.cshp.ca/ advocacy/campaigns/OMAagainstPrescribing_e.asp.

L'Association des pharmaciens du Canada (APhC), la SCPH et le RSP conjoint SCPH-APhC en soins de santé primaires ont brûlé la chandelle par les deux bouts pour formuler un projet intitulé « Access to quality Drug therapy Applying Pharmacists' knowledge to The primary care System (ADAPTS) ", qui s'est mérité une rondelette subvention de huit cent mille dollars de Santé Canada. Le projet ADAPTS mettra à profit les connaissances et les compétences des pharmaciens dans les milieux de soins de santé primaires et mènera à l'exécution de plusieurs actions clés proposées dans le programme de mise en oeuvre du Plan directeur pour la pharmacie. Le projet s'échelonnera de novembre 2009 à mars 2011.

La Société, ses bénévoles et ses employées ont consumé temps et énergie, en 2009, dans d'autres réalisations flamboyantes. La SCPH a nommé de nombreux membres pour la représenter auprès d'organisations externes, incluant le Comité de gestion du projet ADAPTS, le Comité de pilotage du Plan directeur pour la pharmacie, le Corps national d'experts pour la mise en œuvre de la directive nationale concernant l'utilisation des opioïdes et le Comité de planification du Forum canadien 2010 sur la sécurité des patients et l'amélioration de la qualité, parrainé par l'Institut canadien pour la sécurité des patients. Nous avons exécuté une campagne de valorisation sur le rôle du pharmacien d'hôpital dans l'amélioration de la qualité et de la sécurité de l'utilisation des médicaments (http://www.cshp.ca/ advocacy/campaigns/patSafety_e.asp). En collaboration avec la section de l'Alberta de la SCPH, la Société a écrit au doyen de la
Faculté de pharmacie et des sciences pharmaceutiques de l'Université de l'Alberta pour exprimer ses préoccupations à propos du projet de doctorat professionnel en pharmacie de premier cycle et de l'incidence de la formation expérientielle croissante sur les ressources en pharmacie hospitalière. Le personnel de la SCPH a sondé les membres sur les programmes et les services offerts par la Société en vue de la séance de planification stratégique que le Conseil de la SCPH tiendra durant l'été 2010. Nous avons aussi fait part de nos réactions à de multiples parties prenantes : au Comité consultatif sur la santé du ministre de la Santé et du Bien-être de l'Alberta (en collaboration avec la section de l'Alberta de la SCPH, sur les principes et le thèmes qui pourraient guider l'élaboration de la nouvelle législation sur la santé; consultez http://www.cshp.ca/ advocacy/ABhealth_e.asp), au Conseil canadien de l'éducation permanente en pharmacie (sur l'ébauche de la politique sur l'agrément des programmes menant à un certificat), à Santé Canada (sur la Commande électronique de substances désignées [lignes directrices provisoires]) et à l'Institut pour l'utilisation sécuritaire des médicaments du Canada (sur l'ébauche du document concernant la collaboration avec les consommateurs pour prévenir les incidents liés à l'utilisation des médicaments, une stratégie de déclaration et d'apprentissage à l'intention des consommateurs par le truchement du Système canadien de déclaration et de prévention des incidents médicamenteux).

D'intérêt plus immédiat pour les pharmaciens d'établissements, la SCPH a ravivé les clauses de la police d'assurance contre la responsabilité professionnelle qu'elle parraine. C'est ainsi que la couverture s'est vue bonifier du droit de prescrire par les pharmaciens, sans supplément. Cet ajout est entré en vigueur pour la période d'assurance 2009-2010.

Treize ardentes employées ont alimenté le feu pour engendrer ces nombreuses réalisations. Au cours de la dernière année, Robyn Rockwell (agente du service aux membres) a célébré son cinquième anniversaire d'embauche à la SCPH, Laurie Frid (gérante des opérations) a repris ses fonctions après son congé de maternité et son congé parental, Desarae Davidson a été réintégrée dans son poste d'agente des congrès, Colleen Drake a accepté de nouvelles responsabilités comme agente des prix et des RSP, et Julie Maillet s'est jointe à notre équipe comme commis de bureau.

Une année endiablée, sans l'ombre d'un doute! Un cordée de réalisations suffisante pour que la SCPH allume un feu de joie.

Myrella Roy, B. Sc. Phm, Pharm. D., FCCP, est directrice générale de la SCPH. 